\title{
Infrared Small Target Detection Using Modified Order Morphology and Weighted Local Entropy
}

\author{
Xiaocui Zhang ${ }^{a}$, Jiannan Chi ${ }^{b}$, Jingyao Hu, Linna Liu, Yongyue Xing \\ School of Automation and Electrical Engineering, University of Science and Technology Beijing. \\ Beijing 100083, China \\ axc61_zhang@163.com, bsy_jnchi@126.com
}

\begin{abstract}
Keywords: Complex sky background, infrared image, small target detection, order morphology, local entropy.
\end{abstract}

\begin{abstract}
Infrared images in the sky scence often contain different complexity noises, which affect the validity of target detection algorithm and result in high false alarm rate. In order to solve this problem, this paper proposes a method based on weighted order morphology, which utilizes the improved open operation to do the denoising and then uses the edge structuring element to extract the small target, and defines a weighted order morphology-based top-hat (WOTH) operation to suppress background while enhancing the target, and finally complete the detection of target by using a simple adaptive threshold. The algorithm combines three different structuring elements, and has four adjustable parameters. By controlling the structure elements' size and related parameters, the target and the background can be quickly separated, the use of weighted local entropy operation also enhance the target effectively. The experimental results show that the proposed algorithm can effectively suppress different types of interference and accurately detect infrared small targets, and has a good performance in robustness and fastness.
\end{abstract}

\section{Introduction}

Infrared (IR) small target detection is one of the crucial techniques in infrared search and tracking (IRST) systems. Noises contained in the IR images varies from the imaging environments. At the same time, at the long-distance imaging, target in the image usually shows as isolated point or patch, lacks of appropriate structural information, and often be submerged in the noise, thus increases the difficulty of target detection. Therefore, many researchers have paid much attention to the study of IR small-target detection, especially to those images that are against complex and noisy backgrounds.

At present, the main idea of IR small target detection method is target enhancement and background suppression, mainly include the following methods: filter-based method, image decomposition method, human visual based method and morphology method. Filter-based methods are widely used, such as the three-dimensional filter method[1], the maximum and minimum filter , Max-median filter[2], bilateral filter methods[3] and methods combines a variety of filters[4].This methods can be applied to a variety of occasions, but the simplicity and effectiveness of the algorithms conflict with each other. Image decomposition method has also been a lot of attention, this method uses wavelet transform [5], and Fourier transform [6] and so on to the image for time domain analysis or frequency domain analysis. Zhao et al. [7] use the wavelet transform to decompose the image into high-frequency part and low-frequency part, extract and fuse the high-frequency part of the image, then finish the target detection by using threshold segmentation; Bai [8] uses the quaternion to reconstruct the image, obtains the spectrum and amplitude spectrum of the image through the super complex Fourier transform and filters it, and finally returns the time domain to complete the detection task. Such methods usually work well, but are usually computationally complex and time-consuming. In addition, there are recently emerging methods based on human visual, because the target often attracts the majority of human visual attention, so according to the target and the neighborhood contains different amount of information, we can make use of visual contrast mechanism ${ }^{[9]}$ to enhance the target and finish the detection task. For example, Deng et al. ${ }^{[10]}$ Propose a weighted contrast method to suppress the interference while enhancing the target. Han's algorithm ${ }^{[11]}$ reduces the 
computational complexity by using matrix block, and locates the target according to the local contrast mechanism after calculating the visual significance graph. The mathematical morphology method [12] is also a fast and efficient method for small target detection, which utilizes structure elements to filter the target, where the top-hat operation can be used to detect targets less than the structure element, but the algorithm is less effective in dealing with complex noises. In deal with the shortcomings of classical tophat operation, Bai et al.[13] proposed a new tophat operation, which enhanced the effect of target detection, but still had a high false alarm rate when noises shape are similar to target's. Besides, there are large amounts of modified algorithms derived from mathematical morphology, including the toggle contrast operator[14], multiscale center-surround top-hat transform[15], hitormiss transform[16].In addition, there are methods based on vector machines[17], sparse decomposition [18],[19] and so on. However, some methods are computationally intensive and complicated, some algorithms have high false alarm rate when dealing with complex sky background, especially dense clouds, cloud edge and target-like interference.

In this paper, we propose an effective method to detect small targets in complex background. Firstly, we use the improved order morphological operation to suppress the background, remove most of the interference obviously. And then make full use of the difference information between the target and the surrounding neighborhood, by weighting the local information entropy to enhance the target area. Finally, complete the detection of small targets through a simple adaptive threshold processing. In this paper, we use a variety of images with different noises to verify the results of the algorithm. Experiments show that this algorithm can accurately detect the infrared small targets in different complexity scenes, and has good robustness and adaptability.

The remainder of this paper is organized as follows. Section 2 introduces the weighted order morphology-based top-hat (WOTH) method and analyzes parameters. Experiments carried on a set of real infrared images covering typical backgrounds with sky clutters are shown in Section 3. Finally, the paper end ups with a conclusion in Section 4.

\section{The WOTH Small Target Detection Algorithm}

In this section, we introduce a new method for the detection of a small infrared target embedded in cloudy-sky backgrounds with clutters and noise. This method weights the modified local entropy by the improved order-morphology-based top-hat operation to separate the target from jamming objects and subsequently utilizes a simple threshold to detect the target in the weighted result. It is good at suppressing various intricate cloudy-sky backgrounds and improving signal-to-clutter (SCR) values of the image.

\subsection{Background Suppression based on Order-Morphology Tophat}

From the morphological knowledge, the patch structure the image, which is closely related to the size and shape of the structure element, can be extracted by the top-hat operation quickly and easily. However, the classical tophat operation still have high false alarm rate, the reason lies mainly in the core of tophat operations: structure element. First, tophat operation is performed with only one structural element, haven't make full use of the difference information between the target and the neighborhood; Second, in different environments, small target's size is different, fixed structure element's size will be too small or too large to supress various noises, that is, the algorithm has poor adaptability. Therefore, we propose the following OTH operation, by using appropriate size of structure element can reduce a certain amount of calculation, besides, we can adjust the parameters so that the algorithm will has good adaptability to different size of targets.

Define order erosion and order dilation operations as the following formula (1), (2), in which $f(x, y)$ is the original image, $B$ is planar structure element, and $\mu(B)$ is the point count of the structural element $\mathrm{B}$, ord is the order morphology operation ${ }^{[20]}$.

$$
\begin{aligned}
& f \Theta^{n} B=\operatorname{ord}\{n ; I \mid B\}, 0<n<\mu(B) / 2 \\
& f \oplus^{m} B=\operatorname{ord}\{m ; I \mid B\}, \mu(B) / 2<m \leq \mu(B)
\end{aligned}
$$

In the above formula, $n, m$ respectively is erode coefficient and dilate coefficient, collectively referred to as the operating coefficient. The smaller the $n$, the stronger the corrosion;the bigger the 
$m$, the stronger the expansion. $n$ defaults to $1, m$ defaults to $\mu(B) / 2$. On this basis, we define the following operations:

$$
\begin{aligned}
& f \circ_{S S}^{n m} \mathrm{~B}=f(x, y) \ominus^{n} B_{S} \oplus^{m} B_{S} \\
& f \bullet_{M E}^{m n} \mathrm{~B}=f(x, y) \oplus^{m} B_{M} \Theta^{n} B_{E}
\end{aligned}
$$

In the formula (3), $B_{S}$ is a planar structure element, we call $f \circ_{S S}^{n m} \mathrm{~B}$ as order-open operation, which can remove noise less than $B_{S}$. In formula (4),we call $f \cdot \bullet_{M E}^{m n} \mathrm{~B}$ as order-close operation, $B_{M}$ is an edge structure element with inner edge $\mathrm{B}_{\mathrm{Mi}}$ and outer edge $\mathrm{B}_{\mathrm{Mo}}, B_{E}$ is a rectangle structure element, and the size relation is as follows:

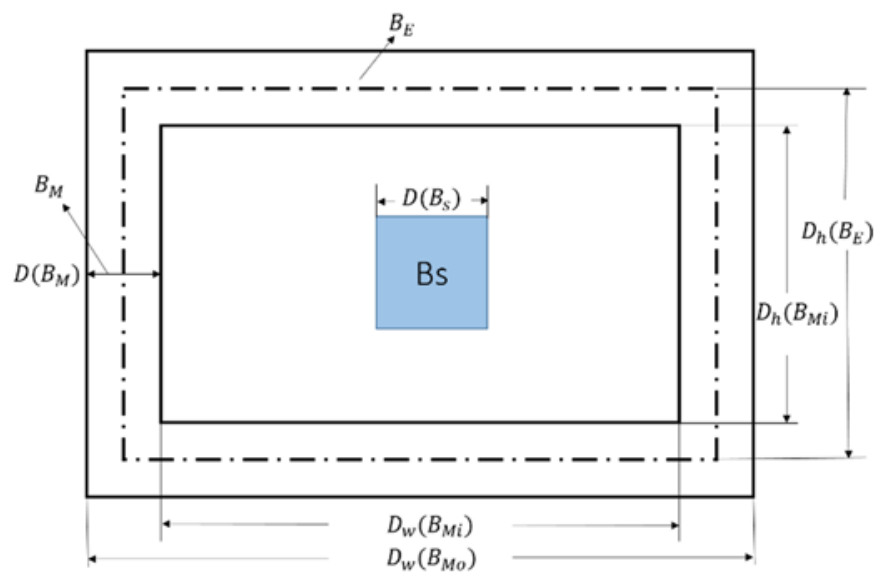

Fig.1 The size of the structuring elements

In Figure $1, B_{S} 、 B_{M i} 、 B_{M o} 、 B_{E}$ are four planar structuring elements, $D(B)$ represents the size of the structure element, $\mathrm{D}\left(B_{M}\right)$ is the width of the edge, and $\mathrm{D}_{w}(B), \mathrm{D}_{h}(B)$ denote the width and height of the structural element $\mathrm{B}$, respectively. $B_{M i} 、 B_{M o}$ Determine the size of the edge structure element $B_{M}$ together, $\mathrm{D}\left(\mathrm{B}_{M i}\right)<\mathrm{D}\left(\mathrm{B}_{M o}\right), \mathrm{D}\left(B_{M}\right)=\left(\mathrm{D}\left(\mathrm{B}_{M o}\right)-\mathrm{D}\left(\mathrm{B}_{M i}\right)\right) / 2$. After defining the above basic operations, we propose OTH operations:

$$
\begin{aligned}
& N(x, y)=I \circ_{S S}^{n m} B \\
& O^{m n}(x, y)=I \bullet m n \\
& O T H=N(x, y)-\min \left[N(x, y), O^{m n}(x, y)\right]
\end{aligned}
$$

In the OTH operation, Eq.(5) is the preprocessing part of the algorithm. This step can not only remove the majority of the noisy noise in the image, but also reduce the amount of subsequent computation. In addition, we can adjust the operation coefficient to achieve the de-noising effect needed by the subsequent algorithm.

In Eq.(6), the $O^{m n}(x, y)$ operation uses the edge structure element to finish the smoothing operation, as shown in Fig. 2,in which the target area is marked by red rectangle, and the noise area is marked by blue elipse. In this step, $\mathrm{D}\left(\mathrm{B}_{M i}\right)$ is slightly larger than the target area, the center pixel area is replaced by the neighborhood area, the information exchange process between the target pixel and the target neighborhood pixel is completed, as Fig.2(b); after that, the order erosion operation will restore the information exchanged in the previous step. Besides, order dilation and order erosion operation also eliminates parts of the positive noises and negative noises, as shown in Fig.2 (c).

Consider the target's intensity is greater than the surrounding background's, after this step, if I (x, $\mathrm{y})$ is the target, its intensity will be down after introducing the neghborhood' slow gray pixel information, then $O^{m n}(x, y)<N(x, y)$; if $\mathrm{I}(\mathrm{x}, \mathrm{y})$ is background, then $O^{m n}(x, y)>N(x, y)$, as shown in Fig. 2 (d). 


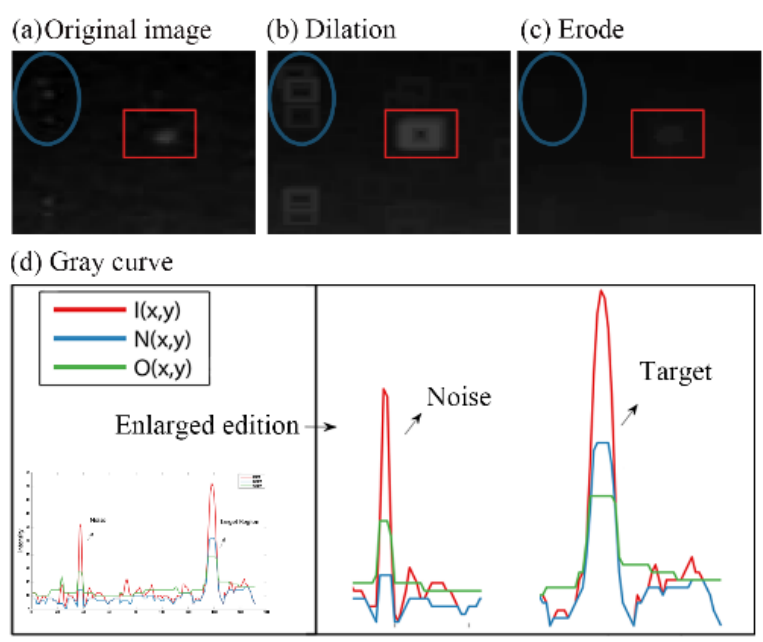

Fig.2 The gray scale of target and noise before and after denoising

Finally, the OTH operation in Eq.(7) has the function of target enhancement and background suppression. If $N(x, y)<O^{m n}(x, y)$, then $O T H(x, y)=N(x, y)-N(x, y)=0$, this area is noise area and filter it off; otherwise, $\operatorname{OTH}(x, y)=N(x, y)-O^{m n}(x, y)$,this area is target and retained, but the gray value of target is reduced.

\subsection{Target Enhancement based on WOTH}

After the background suppression with OTH, most of the noise in the image has been filtered, especially filtered the edge noises that will easily influence the denoising result. However, the target intensity is also weakened while removing the noises, which is not conducive to the subsequent processing.

Noticed that a small target usually emerges as a small bright region in an infrared image, such target can be isolated from the background based on the difference of gray values between the target and background, and information entropy is just an index that can reflect and emphasize the differences information. The local entropy operator is expressed as follows ${ }^{[21]}$ :

$$
W(x, y)=-\sum_{i=1}^{m_{i}}\left(f_{i}-\bar{f}\right)^{2} p_{i} \log _{2} p_{i} \text {, where } p_{i}=\frac{n_{i}}{M \times N}
$$

Where $\mathrm{M} \times \mathrm{N}$ is the size of the neighboring area $\mathrm{S}, \mathrm{M}$ and $\mathrm{N}$ are odd integer numbers, respectively; $p_{i}$, the probability of i'th gray value $f_{i}$ occurring in set $\mathrm{S}$; and $\bar{f}$ is the mean of gray values in set $S$. In this paper, we set the value $M=N=2$.

Local information entropy is used to represent the amount of information in the local region. When the region is flat, the entropy is very small, and when the intensity of the region changes sharply, the entropy value is high, such as the small target area, the edge area and so on. Therefore, the local information entropy can suppress gentle change area in the background, including speckle patches that are similar to the shape and structure of the target in the region, but in some isolated noise patches, the entropy is still large. The OTH operations smooth out isolated speckle patches, but cannot remove the target noise hidden in the clouds. Weighted OTH (WOTH) operation is used to combine the advantages of OTH and local entropy to strengthen the target, and to compensate the disadvantages of the two to suppress the background noise.

The weighted OTH operation is defined as follows:

$W O T H=W(x, y) * O T H(x, y)$

Where $\mathrm{W}(\mathrm{x}, \mathrm{y})$ and $\mathrm{OTH}(\mathrm{x}, \mathrm{y})$ denote the local entropy and the OTH operation result at the point $(\mathrm{x}, \mathrm{y})$.

\subsection{Target Detection Method based on WOTH}

Above, the background suppression and target enhancement are completed, and then the target detection can be finished by simple thresholding. The threshold Th is defined as follows:

$T h=0.7 * \max (O T H(x, y))$

In equation (10), max represents the maximum value of the image OTH (x, y).

The overall algorithm flow chart is shown below: 


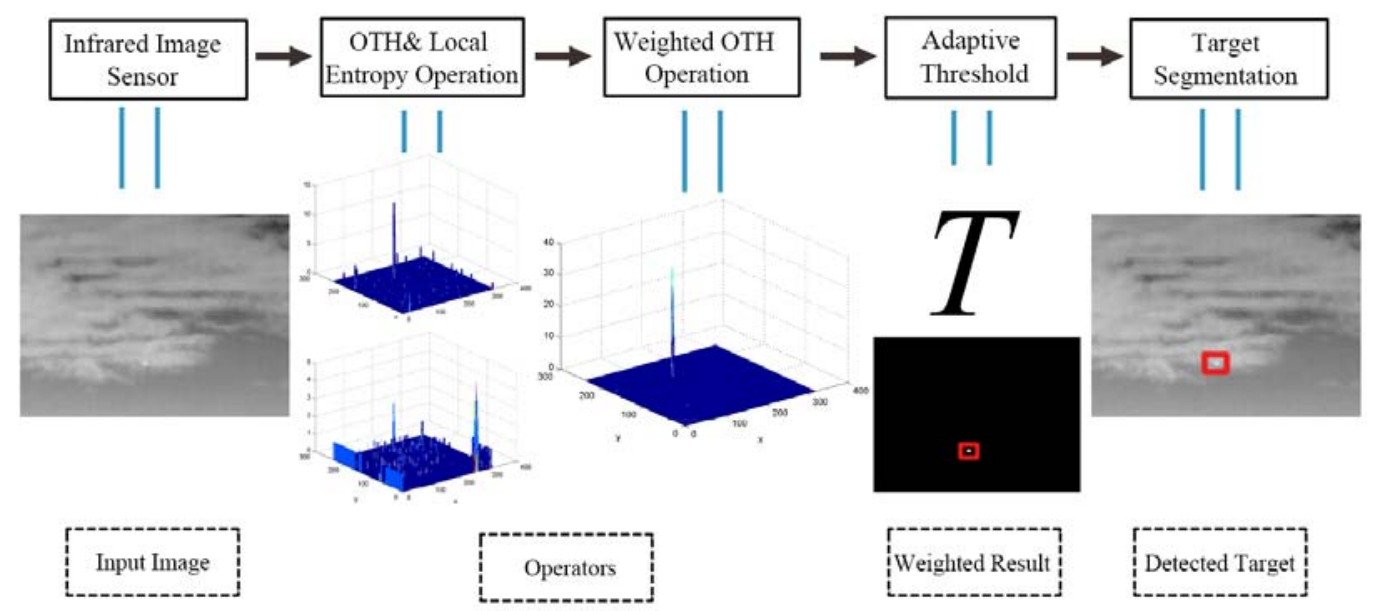

\subsection{Parameter Analysis}

Fig.3 Proposed small target detection system

Preprocessing can remove most of the simple noise and reduce the computational complexity .The preprocessing effect is related to the structure element $B_{S}$ 's size, the size relationship of the structure element $D\left(B_{S}\right)$, the target $D(T)$ and the noise $\mathrm{D}(\mathrm{N})$ should satisfy $D(N)<D\left(B_{D}\right)<D(T)$.

In the equation $N(x, y)=I \circ \circ_{S S}^{n m} B$, the selection of operation coefficients $n$ and $m$ also affects the results of the algorithm. According to the order morphology, the smaller the $n$ and $m$, the stronger the smoothing ability of the image to the bright noise, and the smaller the value of $\mathrm{N}(\mathrm{x}, \mathrm{y})$. However, the stronger erosion can lead to the loss of image information. In addition, the greater the $B_{s}$, the stronger the algothrim's denoising capability;but increase the size of $B_{s}$ would also decrease the target intensity, so that the noise smoothing capability can be improved by adjusting the $n$ and $m$ parameters without changing the size of $B_{s}$. In the morphological opening operation, the size of the noise and structure element and target should be in ascending order, in this way, the target can be retained and the noise can be removed, as shown in Fig.4a. The target will be filtered out when the structure element is the same as the target size or larger than the target, as shown in Fig.4b. In the OTH operation, by adjusting the parameters $n$ and $m$ of the large structure elements, we can achieve the same denoising effect that is achieved by using small structure elements, such as Fig.4c and Fig.4d. On the basis of retaining the target, it has better smoothing effect.

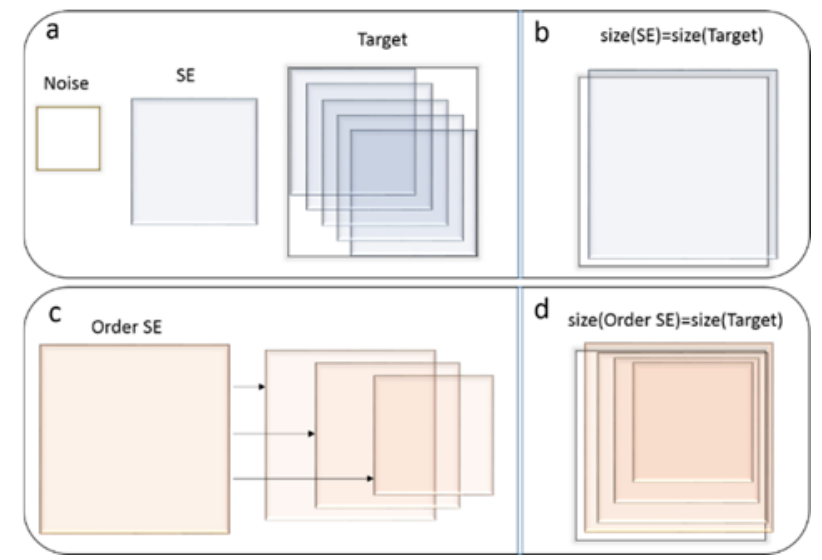

Fig.4 The size of the structure element

In figure 5,(a),(b) and (c) are the results of adopting different size structure elements and different $n$ and $m$ parameters. Fig.5(d) shows the gray curve of three figures along the red line's horizontal direction, where curve A,B and C respectively correspond to A, B and C target area. As can be seen from the graph, after denoising with large structuring elements, at point $\mathrm{C}, N(x, y)<O^{m n}(x, y)$, which results in at the subsequent OTH operation, the target portion at $\mathrm{C}$ is treated as noise and will be eliminated. In the Fig.5(b), by adjusting the $n$ and $m$ parameters, not only more target information is retained, but the target gray level is maintained without affecting the extent of subsequent algorithm, at the same time, the noise marked as $\mathrm{M}$ is also eliminated by this way. 


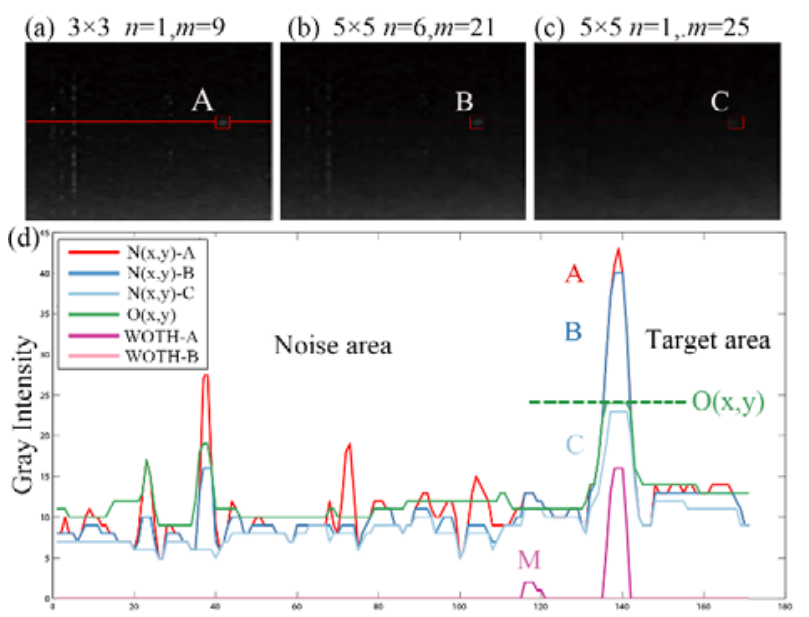

Fig. 5 The relationship between $\mathrm{B}_{\mathrm{s}}$ and parameter $n 、 m$.

In $O^{m n}(x, y)$ operations, we select rectangle that is similar to the target shape as the basic structure element shape, and select the structure element which is slightly larger than the target. However, unlike classical open operations, the algorithm is not sensitive to the size of the structure elements. Because the final result of the OTH operation not only depends on the $O^{m n}(x, y)$ operation, but also depends on the $\mathrm{N}(\mathrm{x}, \mathrm{y})$ operations, the two are pinned down each other, thus reducing the sensitivity of the algorithm to the structural elements, and improving the robustness of the algorithm.

In the $O^{m n}(x, y)$ operation, the edge structure element $\mathrm{B}_{\mathrm{M}}$ is used to dilate the image weakly, and then the $B_{E}$ is used to do weak erode operation, which is equivalent to the open operation of the image to some extent. According to the nature of open operation, we should keep the size of structure elements that is used to carry the dilation and erosion operation the same, which means, $D\left(B_{E}\right)=$ $\mathrm{D}\left(\mathrm{B}_{\mathrm{Mo}}\right)$,so that the target area will remain the same. In addition, $\mathrm{D}\left(\mathrm{B}_{\mathrm{Mi}}\right)$ should be larger than the target area so that the algorithm can detect the target completely.

The denoising capability of the algorithm is also related to the width of $D\left(B_{M}\right)$. The larger the $D\left(B_{M}\right)$, the more the noise is fused with the surrounding pixels, and the more passive noise is finally introduced, thus results in a reduction in algorithm's denoising ability. Figure 6 shows the effect of using different sizes $\mathrm{D}\left(B_{M}\right)$ on $O^{m n}(x, y)$ operation, using the partial noise as Fig.6(b) in the image (a) as an example. In the first column to the third column, $D\left(B_{M}\right)=1, D\left(B_{M}\right)=2$ and $D\left(B_{M}\right)=3$ respectively. The first row is the result of the dilation operation using the $\mathrm{B}_{\mathrm{M}}$ structural element, and the second is the result of $O^{m n}(x, y)$ operation that is erosion result after dilation. As is shown, the larger the $\mathrm{D}\left(B_{M}\right)$ is, the more pixels are involved in the fusion of information, and the more residual noise. In the algorithm, the general set $\mathrm{D}\left(B_{M}\right)=1$.

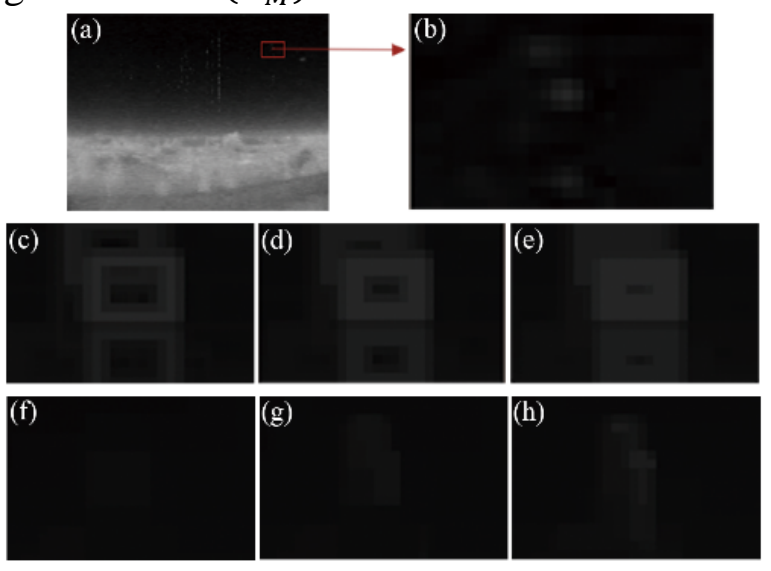

Fig.6 The relationship between $\mathrm{D}\left(B_{M}\right)$ and denoising ability 


\section{Experimental Results and Analysis}

In order to verify the effectiveness of the algorithm, we selected some images with different interference types from typical infrared sequence images, and divided them into six categories: simple cloud interference, bright light and halo noise interference, high-rise buildings interference, large cloud interference, complex cloud edge interference, dense clouds and target-like noise interference. Six representative images are selected as shown in Figure 6 (a1) to (a6). In these images, the complexity of the background noise increases gradually, the cloud noise gradually increases, and the relative position of the small target and the cloud is different. In addition, the above pictures also contain the interference spots that is similar to small target, which incerase the difficulity of target detection.

All experimental images are 8-bit JPEG-type grayscale, with the size of $320 * 240$ pixels. All experiments in this paper are simulated using MATLAB®R2012a, under the WIN7 platform with the Intel (R) Pentium (R) P6200 processor.

In the experiment, we use the three dimensions (3D) gray scale diagram to visually represent the gray distribution in the image and introduce the SCR ${ }^{[21]}$ parameter. The SCR parameter can be used to evaluate the difficulty of target detection in an image. The smaller the value, the more difficult it is to detect the target. The definitions is as follows:

$$
\operatorname{SCR}=\frac{\left|\mu_{t}-\mu_{c}\right|}{\sigma_{c}}
$$

In which, $\mu_{t}$ and $\mu_{c}$ are the mean value of the target region and the background region, respectively. $\sigma_{c}$ is the standard deviation of the background region.

\subsection{The Detection Results of the WOTH Algorithm}

WOTH algorithm is used for target detection of the images mentioned above, and the result is shown in figure 7. In Fig.7, (a1) to (a6) are original images, (b1) to (b6) are the 3D grayscale maps of the original image, and (c1) to (c6) represent the 3D grayscale maps after the background suppression using the algorithm, and (d1) to (d6) represent the final detection results. The target section in the image is marked with a red rectangle. Experimental results show that the algorithm has good effect on sky-background with different noise levels, and the algorithm has good robustness regardless of different background complexity.
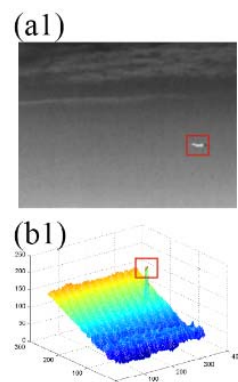

(c1)

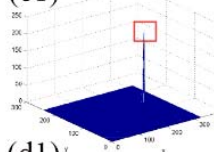

(d1)

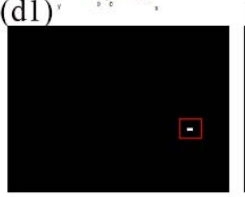

(a2)
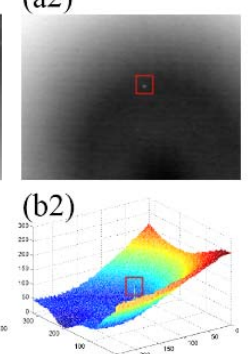

(c2)

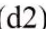

(d2)

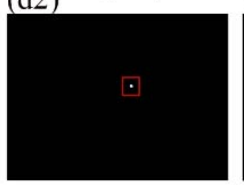

(a3)

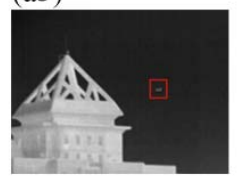

(b3)

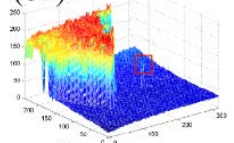

(c3)

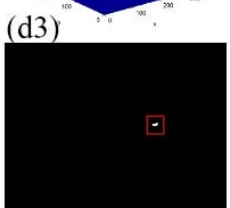

(a4)

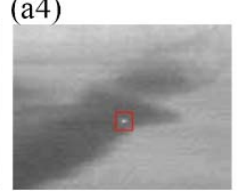

(b4)

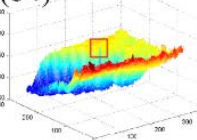

(c4)

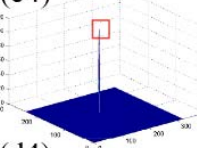

(d4)

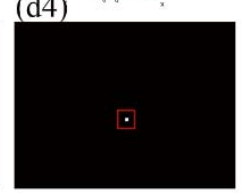

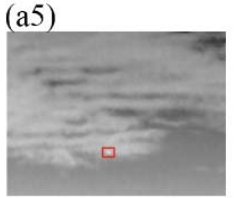

(b5)

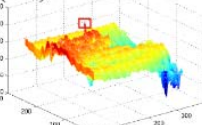

(c5)

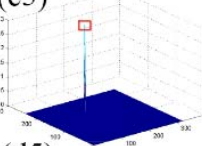

(d5)

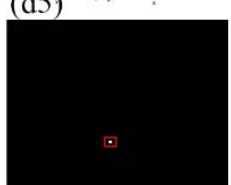

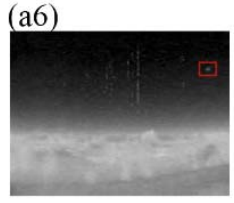

(b6)

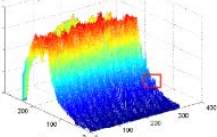

(c6)

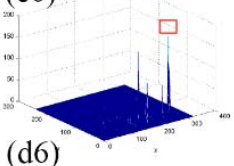

(d6)

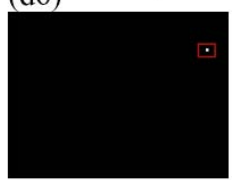

Fig.7 WOTH operation on different images of the target detection results

In addition, from the above we can see that the algorithm does not require highily for the rectangle structure element $\mathrm{B}_{\mathrm{M}}$ 's size, so long as the structure element is larger than the target. Figure 8 shows the change in the SCR value of the output image when the size of the structural element $B_{M}$ changes. In Fig.8, (a) and (b) show the (a4) and (a5) images' final output SCR value's trend graph , which shows the result of adjusting the size of the structural element $B_{M}$,both the (a4) and (a5) images have complex background noises. It can be seen that adjust the size of the rectangle will affect the output 
of the SCR value, but the algorithm still maintains a good output effect compared with the original SCR value.
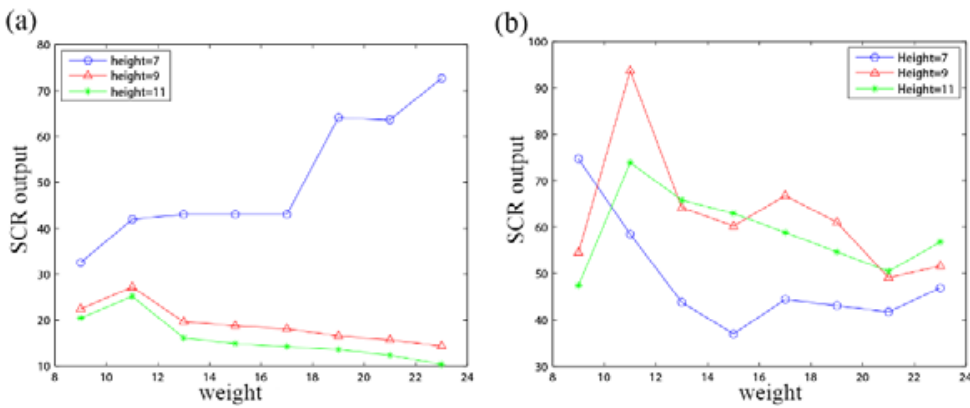

Fig.8 SCR value of the output image when the $B_{M}$ size changes

\subsection{Comparison Results of This Algorithm with other Algorithms}

We use the method of image decomposition, human visual method and morphological method introduced in the introduction as the contrast method to verify the performance of the WOTH algorithm. The algorithms used to compare are Bai's algorithm ${ }^{[8]}$, Han's algorithm ${ }^{[11]}$, and NWTH algorithm ${ }^{[13]}$.Figure 9 shows the results of the comparison, from top to bottom five rows, respectively, said the original images, result of using Bai's algorithm, Han 'algorithm, NWTH algorithm and the results of our WOTH algorithm, columns 1 6 were 6 different experimental images respectively. In the figure, the target is marked with red rectangle, and the noise is marked with red circle. The results show that the four algorithms both have good detection results for infrared images with mild background variations, such as column 1 and column 4; but when the background has bigger gray intensity fluctuation, especially the edge is more, the false alarm rate of Bai's algorithm is higher, and it can't distinguish the edge region and the target well, such as columns 3, 5, 6; In addition, Han's algorithm cannot distinguish between noise spots and targets, and the effect is poor when there are noise spots similar to target in the background. The NWTH algorithm has good robustness to most of the noise, but when the background is complex and the gray scale is more complex, the anti-noise performance is poor, and there is still some false alarm rate. The WOTH algorithm performs well both in complex dense backgrounds and in images with interference noises similar to the target.
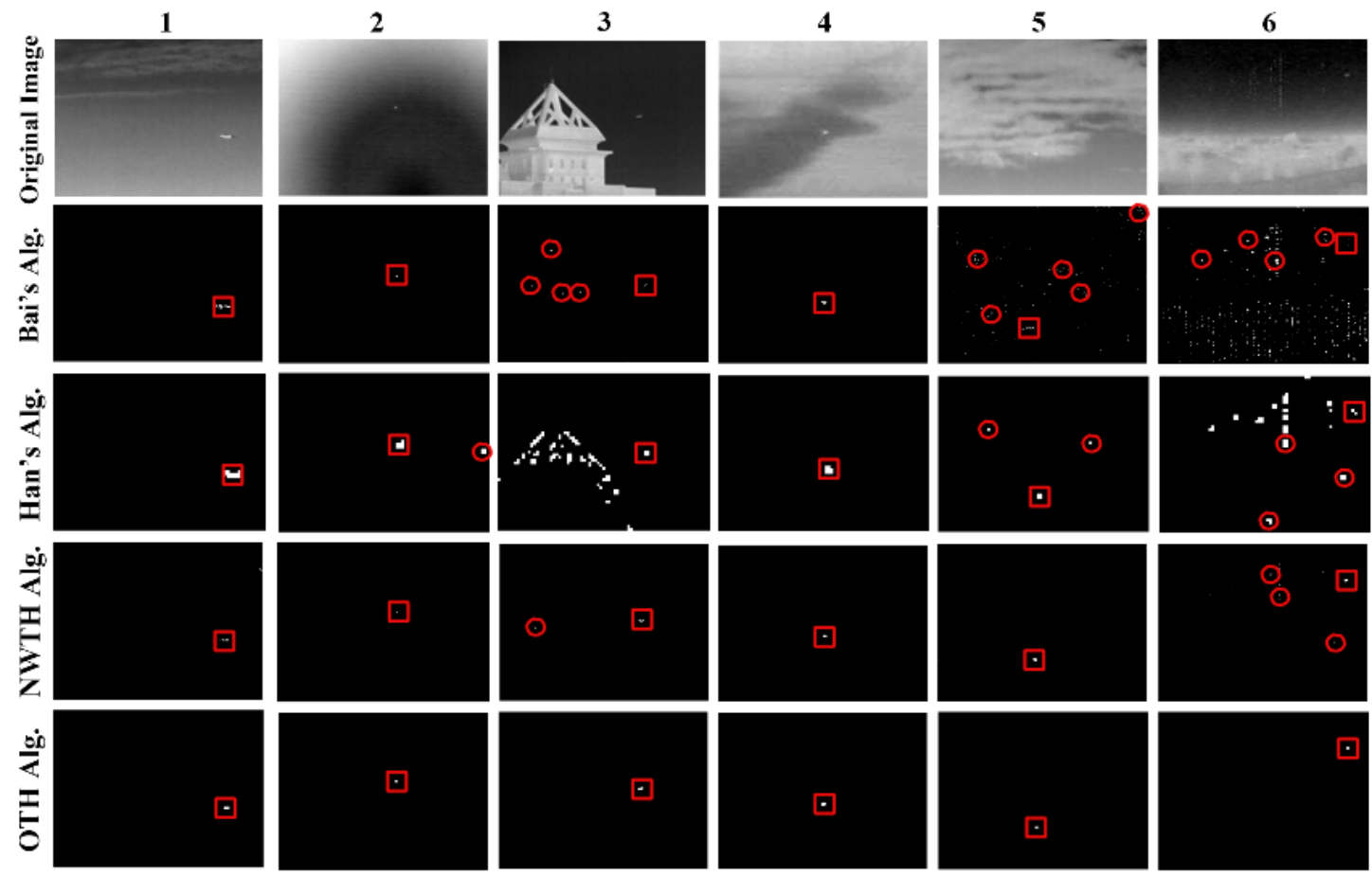

Fig.9 Small IR target detection for images captured in various scenes with different algorithm

Figure 9 intuitively reflects the four kinds of algorithms's effort on the the interference suppression, in which the algorithm of Bai and Han is not effective when there are complex background and noise interference to the target, but the NWTH algorithm performs poorly only when there are multiple 
levels of cloud and target-like noise interference in the background. To further compare the results of WOTH algorithm with NWTH, the SCR values of the two algorithms are compared. The results are shown in Fig 10. Three histograms of different colors represent the original SCR value, the SCR value after the NWTH algorithm, and the SCR output value after using the algorithm, and the abscissa is used to represent the 6 images used in the experiment. It can be seen from the figure that the background suppression effect of WOTH algorithm is much better than that of NWTH algorithm, especially when dealing with complex noise, the WOTH algorithm is more effective, and has greatly increases the SCR value of the image and suppresses almost all the noise, which has good robustness and superior performance.

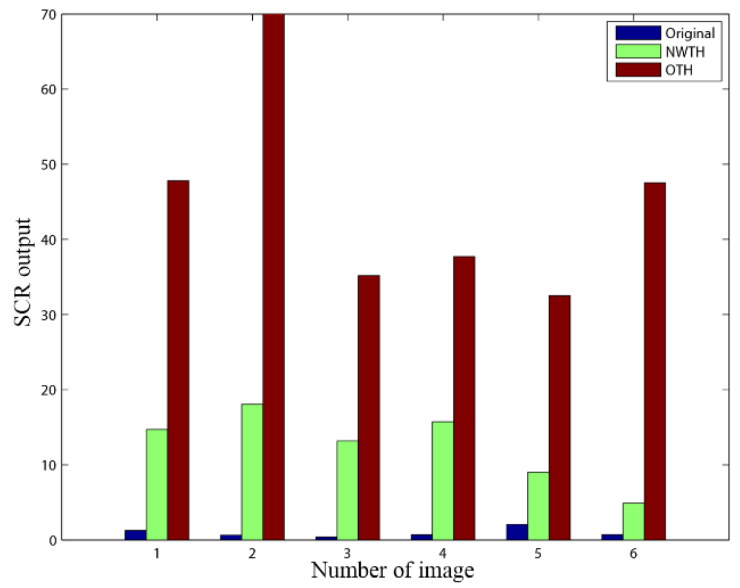

Fig.10 Comparison of SCR_output between OTH and NWTH

Finally, in order to verify the rapidity of the algorithm, we calculate the average computing time of the four algorithms, and the results are as Table 1:

Table 1 . The average calculation time of each algorithm

\begin{tabular}{ccccc}
\hline & Han’s Algorithm & Bai’s Algorithm & NWTH Algorithm & WOTH Algorithm \\
\hline Computing time(s) & 0.388065 & 0.319518 & 0.027646 & 0.067254 \\
\hline
\end{tabular}

From the above experiments we can conclude that the NWTH algorithm and our algorithm have the fastest computing time, but the NWTH algorithm does not work well with complex background. The Bai's algorithm works well in dealing with the background noise of a gentle change, but cannot handle a background with large local gradations. Han's algorithm can maintain the contrast between the target and the surrounding neighborhood, but it is poor in robustness when it faces the interference with the target gray scale. The algorithm proposed in this paper can not only realize fast detection, but also perform well in the face of different noises.

\section{Conclusion}

In this paper, we introduce a small target detection method in infrared images based on weighted order morphology top-hat operation, which make use of the merit of order morphology and the local entropy. The proposed algorithm can work well under different kinds of backgrounds. The experiment results demonstrate that the proposed method can achieve better accuracy and efficiency performance compared with state-of-the-art methods.

\section{References}

[1]. Liu X. and Zuo Z. A Dim Small Infrared Moving Target Detection Algorithm Based on Improved Three-Dimensional Directional Filtering .Communications in Computer and Information Science, vol. 363.New York, NY, USA: Springer-Verlag, 2013, ch. 13, p. 102-108.

[2]. Venkateswarlu R. Max-mean and max-median filters for detection of small targets [J]. Proceedings of SPIE - The International Society for Optical Engineering, 1999, 3809, p.74-83. 
[3]. Bae T W, Kim H G. Adaptive Bilateral Filter for Infrared Small Target Enhancement [M]. Proceedings of Second International Conference on Electrical Systems, Technology and Information 2015 (ICESTI 2015). Springer Singapore, 2016.

[4]. Wan M J, Gu G h, Cao E, et al. In-frame and inter-frame information based infrared moving small target detection under complex cloud backgrounds [J]. Infrared Physics \& Technology, 2016, 76, p.455-467.

[5]. Wei Y, Shi Z L. An automatic target detection algorithm based on wavelet analysis for infrared image small target in background of sea and sky [J]. Proceedings of SPIE - The International Society for Optical Engineering, 2003, p.123-131.

[6]. Qi S , Ma J, Li H, et al. Infrared small target enhancement via phase spectrum of Quaternion Fourier Transform[J]. Infrared Physics \& Technology, 2014, 62(2), p.50-58.

[7]. Zhao J, Liu F, Mo B. An Algorithm of Dim and Small Target Detection Based on Wavelet Transform and Image Fusion.The 2012 Fifth International Symposium on Computational Intelligence and Design. Washington, DC, USA, 2012, 2(4),p.43-45.

[8]. Bai T, Tian J W, Sun X. A robust method for infrared small target based on saliency detection. Eighth International Conference on Digital Image Processing. Chengdu, China, 2016, p.100330O.

[9]. Cui Z, Yang J, Jiang S, et al. An infrared small target detection algorithm based on high-speed local contrast method [J]. Infrared Physics \& Technology, 2016, 76, p.474-481.

[10]. Deng H, Sun X, Liu M, et al. Small Infrared Target Detection Based on Weighted Local Difference Measure [J]. IEEE Transactions on Geoscience \& Remote Sensing, 2016, 54(7), p.4204-4241.

[11]. Han J, Ma Y, Zhou B, et al. A Robust Infrared Small Target Detection Algorithm Based on Human Visual System [J]. Geoscience \& Remote Sensing Letters IEEE, 2014, 11(12), p.21682172.

[12]. Zhao J, Feng $\mathrm{H}, \mathrm{Xu} \mathrm{Z}$, et al. Real-time automatic small target detection using saliency extraction and morphological theory[J]. Optics \& Laser Technology, 2013, 47(4),p.268-277.

[13]. Bai X, Zhou F. Analysis of new top-hat transformation and the application for infrared dim small target detection[J]. Pattern Recognition, 2010, 43(6),p.2145-2156.

[14]. Mendiola-Santibanez J D, Terol-Villalobos I R. Filtering of mixed Gaussian and impulsive noise using morphological contrast detectors[J]. Iet Image Processing, 2013, 8(8),p.131-141.

[15]. X. Bai, F. Zhou, B. Xue, Fusion of infrared and visual images through region extraction by using multi scale center-surround top-hat transform, Opt. Express 19 (9) (2011) ,p.8444-8457.

[16]. Murray, Paul. Extensions of the hit-or-miss transform for feature detection in noisy images and a novel design tool for estimating its parameters[D]. University of Strathclyde, 2012.

[17]. Zhai S, Jiang T. Sparse representation-based feature extraction combined with support vector machine for sense-through- foliage target detection and recognition[J]. Iet Signal Processing, 2013, 8(5),p.458-466.

[18]. Gao C, Meng D, Yang Y, et al. Infrared patch-image model for small target detection in a single image[J]. IEEE Transactions on Image Processing A Publication of the IEEE Signal Processing Society, 2013, 22(12),p.4996-5009.

[19]. Zhang X, Ren K, Gao J, et al. Infrared moving small target detection based on saliency extraction and image sparse representation.International Symposium on Optoelectronic Technology and Application. Beijing, China.2016,p.1015734.

[20]. Chi J N, Fu P F P, Wang D S, et al. A detection method of infrared image small target based on order morphology transformation and image entropy difference[J]. 2005, 8,p.5111-5116.

[21]. Deng H, Sun X, Liu M, et al. Small Infrared Target Detection Based on Weighted Local Difference Measure[J]. IEEE Transactions on Geoscience \& Remote Sensing, 2016, 54(7),p.4204-4214. 